\title{
Wide-angle reflection-mode spatial filtering and splitting with photonic crystal gratings and single-layer rod gratings
}

\author{
Andriy E. Serebryannikov,,${ }^{1,2,5}$ Philippe Lalanne, ${ }^{3}$ Alexander Yu. Petrov, ${ }^{4}$ and Ekmel Ozbay ${ }^{2}$ \\ ${ }^{1}$ Faculty of Physics, Adam Mickiewicz University, 61-614 Poznań, Poland \\ ${ }^{2}$ Nanotechnology Research Center-NANOTAM, Bilkent University, 06800 Ankara, Turkey \\ ${ }^{3}$ Laboratoire Photonique Numérique et Nanosciences, Institut d'Optique d'Aquitaine, Université de Bordeaux, \\ CNRS, 33405 Talence, France \\ ${ }^{4}$ Institute of Optical and Electronic Materials, Hamburg University of Technology, 21073 Hamburg, Germany \\ ${ }^{5}$ e-mail: andriy@bilkent.edu.tr
}

Received August 11, 2014; accepted September 14, 2014;

posted September 29, 2014 (Doc. ID 220636); published October 22, 2014

\begin{abstract}
New diffractive optical elements offering a frequency tolerant, very efficient, high-pass and bandpass spatial filtering over a broad range of incidence angles are demonstrated by numerical simulations. The device operates in reflection mode owing to the (nearly) perfect blazing. It relies on two-dimensional square-lattice photonic crystals composed of dielectric rods with simple corrugations at the interface. Similar performance can be obtained with gratings composed of a single rod layer placed in the near field of a metal mirror, indicating a route to geometries that can be easily fabricated with modern nanotechnologies. Also equal splitting between zero and first negative orders can be obtained for incidence-angle variations that are wider than $60^{\circ}$. (c) 2014 Optical Society of America

OCIS codes: (050.1950) Diffraction gratings; (070.6110) Spatial filtering; (160.5298) Photonic crystals; (050.1940) Diffraction.
\end{abstract}

http://dx.doi.org/10.1364/OL.39.006193

Spatial (angular) filters represent analogues of conventional frequency filters that show a similar behavior but in the incidence angle domain, while the frequency is fixed. Thus, they can also be considered from the spatial-frequency filtering perspective [1]. Various spatial filters were suggested that are based on artificial anisotropic media [2], interference patterns [1], photonic crystals (PhCs) [프-6], metallic grids []], (non-) resonant grating systems [ $\underline{-11}$ ], multilayer stacks with a prism [12], and axisymmetric photonic microstructures [13].

The known mechanisms of spatial filtering include those using $[\underline{2}-\underline{5}]$ or not using $[\underline{1}, \underline{8}]$ specific dispersion features. The former can give more freedom in design, because the required features can remain within rather wide ranges of variation in frequency and/or angle of incidence. For instance, low-pass spatial filtering can be obtained by using isotropic-type dispersion, which corresponds to refractive index $0<n<1$ [14]. In turn, highpass and bandpass filtering requires anisotropic-type dispersion. Generally, that can be achieved by using anticutoff media [2], which is often associated with hyperbolic metamaterials [15] or PhCs with the corresponding dispersion type that enables blocking transmission in the vicinity of zero tangential wavenumber [3-5]. The problem usually appears regarding the possibility of obtaining the same, desirably perfect, i.e., $100 \%$ efficiency within the entire wide pass and stop angular bands of transmission [9-11]. High-pass spatial filtering that matches this condition has been demonstrated in transmission mode in a slab of the two-dimensional, squarelattice $\mathrm{PhC}$ [3]. For operation in reflection mode, high-pass and bandpass spatial filters offering high efficiencies over broad angle intervals are required but have not been demonstrated yet. It is worth noting that designing spatial filters in reflection mode is much more difficult. In transmission, zero-order gratings may be used to redistribute the incident energy between two channels, the zero reflected and transmitted orders. In contrast, redistribution between zero and one of higher orders is required in a pure reflection mode. Hence, reflection mode spatial filtering is closely related to the blazing that is a classical problem of diffraction gratings.

The blazing effect is probably the most important property of diffraction gratings. The structures enabling it have been studied in both frequency and incidence angle domains. Blazing at a single wavelength for a specific angle of incidence has been obtained in various structures. The difficulty arises when it should be performed over a broad spectral [16] or incidence angle [17] interval. The studies related to splitting [18] and the Littrow mount regime [19] should be mentioned in this concern. However, the earlier studies do not indicate a route to the (nearly) perfect, frequency tolerant, wide-angle spatial filtering. Note that energy redistribution between two channels that may appear due to either different diffraction orders or different polarizations (or both) is a common feature of spatial filtering and splitting $[\underline{18}, \underline{20}]$.

In this Letter, we demonstrate that the wide-angle high-/band pass, (nearly) perfect spatial filtering can be obtained in reflection mode in the slabs of the squarelattice rod-type PhCs with the corrugated interface layer, which are known as $\mathrm{PhC}$ gratings. A variety of the regimes of the diffraction-inspired asymmetric transmission arising in PhC gratings has recently been studied that are connected with blazing $[21,22]$. Here, we suggest $\mathrm{PhC}$ gratings that enable the (nearly) perfect, wideband blazing in reflection mode and steep switching between zero and the first negative orders that are required for spatial filtering. Then, it will be shown that the regular (noncorrugated) part of the $\mathrm{PhC}$ grating can be replaced 
with a metallic reflector, indicating a route to compact performances that are based on the single-layer rod gratings. In addition, we show that a parameter adjustment can result in the nearly perfect wide-angle splitting in the $\mathrm{PhC}$ gratings, which are also appropriate for spatial filtering. Finally, problems related to the splitting regime are discussed. The presented results are obtained by using the original coupled-integral-equations technique and MATLAB code based on fast iterative solution of the simultaneous integral equations in frequency domain by using pre-conditioning [23]. It has controllable accuracy and convergence and is quite flexible regarding geometry of the studied periodic structure. The long-time experience of using this technique enables a well-justified choice of discretization parameters. To obtain each reflection map presented below, simulations were performed for $1.2 \times 10^{5}$ frequency-angle pairs.

The studied structures are assumed to be infinitely extended and periodic along the corrugations and illuminated by the $s$-polarized plane wave with an electric field vector that is parallel to the rod axes, at the angle $\theta$. Figure 1 shows the geometry and reflection results for a $\mathrm{PhC}$ grating composed of the circular rods with diameter $d$ and permittivity $\varepsilon_{r}$. The total number of the rod layers here is $N=12$. The corrugations are obtained by removing every second rod from the interface layer of the noncorrugated slab of square-lattice $\mathrm{PhC}$ with the lattice constant $a$. Thus, the grating period is $L=2 a$. Geometrical and material parameters have been chosen that are either the same as or similar to the $\mathrm{PhC}$ gratings from our earlier studies [21,22].

For the (nearly) perfect, frequency tolerant, wideband spatial filtering, a large area in the $(k L, \theta)$-plane is required $(k=\omega / c$ is free-space wavenumber, $\omega=2 \pi f)$, for which the -1 -order efficiency $r_{-1} \approx 1$, while $r_{0} \approx 0$. One can see in Fig. 1(b) that a proper parameter adjustment enables the desired behavior of $r_{-1}$, e.g., $r_{-1}>0.95$
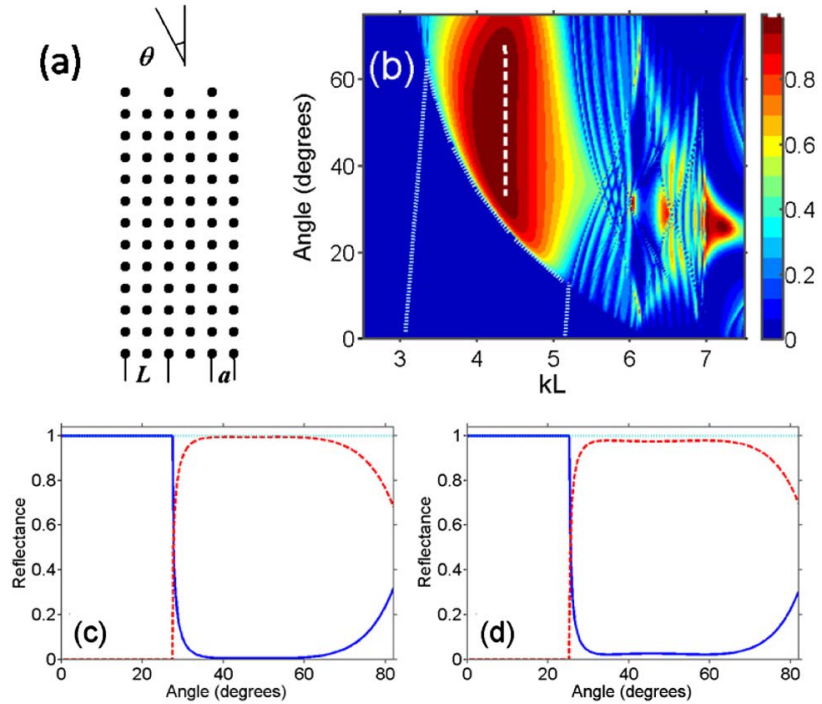

Fig. 1. (a) Geometry of the PhC grating $(L=2 a)$. (b) $r_{-1}$ in the $(k L, \theta)$-plane at $d / a=0.45$ and $\varepsilon_{r}=9.61$. Dashed line, angleindependent regime of $r_{-1} \approx 1$; dotted lines, approximate boundary of the stop band in which $r_{0} \approx 1$. (c), (d) $r_{0}$ (solid line), $r_{-1}$ (dashed line), and $R=r_{0}+r_{-1}$ (dotted line) versus $\theta$ at (c) $k L=4.3$ and (d) $k L=4.4$. at least for $31^{\circ}<\theta<70^{\circ}$ when $k L=4.3$. Accordingly, the diffraction angle $\phi_{-1}$ varies nearly from $-70^{\circ}$ to $-31^{\circ}$. Once the order $m=-1$ starts propagating, it takes almost all the incident wave energy, i.e., there is just a narrow angle interval between $R=r_{0}=1$ and $R=r_{-1} \approx 1$. Hence, low-pass and high-pass spatial filters can be obtained in the same configuration at a fixed frequency.

To better demonstrate the switching in the $\theta$ domain, Figs. $1(\mathrm{c})$ and $1(\mathrm{~d})$ present $r_{0}$ and $r_{-1}$ versus $\theta$ at the two selected values of $k L$. It can be seen that the perfect blaze can be obtained in a very wide $\theta$ range, whereas zero order, which may propagate at any $k L$ and $\theta$ values, is well suppressed. The reflected first negative order now plays the same role as the transmitted zero order in the transmission mode spatial filters in slabs of noncorrugated PhCs [3]. However, the direction of the outgoing wave now depends on $\theta$ in a more complex fashion. Note that the ranges where spatial filtering appears in the studied structure include the Littrow mount regime, for which $2 L \sin \theta=-m \lambda$ and $\theta=-\phi_{m}$. Therefore, it could be stated that high-pass spatial filtering appears due to the order $m=-1$ in a wide vicinity of this regime.

Next, let us investigate whether high-/band pass filtering and wide-angle splitting with $r_{0}=r_{-1}=0.5$ can be obtained in the same structure. Figure 2 presents an example for the $\mathrm{PhC}$ grating, which differs from that in Fig. 1 only for the values of $d / a$ and $\varepsilon_{r}$. As can be seen in Fig. 2(b), $r_{0} \approx r_{-1} \approx 0.5$ in a wide range of $\theta$ variation, on the right of the region of $r_{-1} \approx 1$, i.e., in the vicinity of $k L=5.6$. This peculiar case is associated with the isolines of $r_{0}$ and $r_{-1}$ (not shown) that are obtained at fixed frequency and parallel to $\theta$ axis. The obtained results show that the splitting with $r_{0} \approx r_{-1} \approx 0.5$ and spatial filtering regime with $r_{-1} \approx 1$ can co-exist in one configuration. In Fig. 2(b), high-pass spatial filtering occurs at $k L=4.9$. Multiple diffraction anomalies and
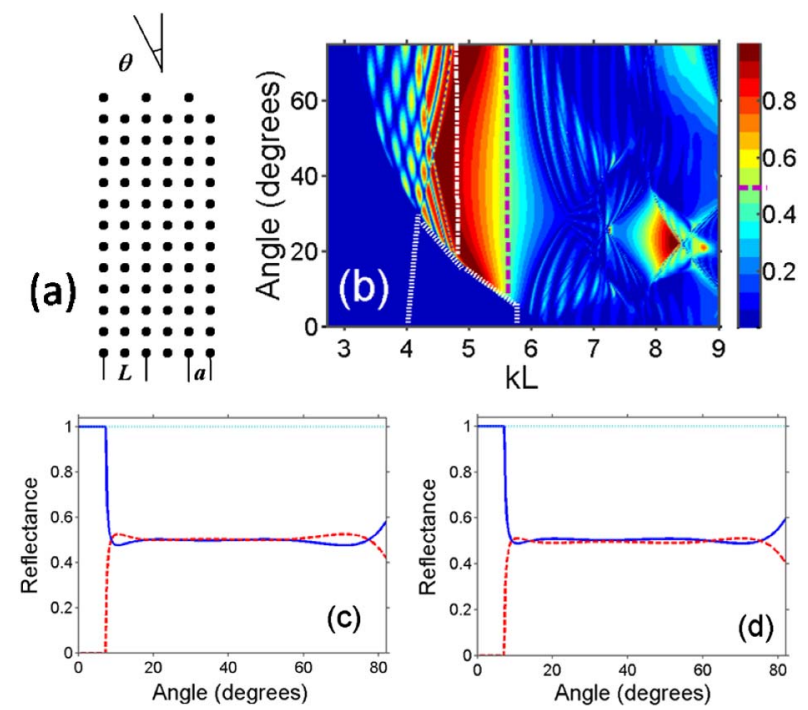

Fig. 2. (a) Geometry of the PhC grating $(L=2 a)$; (b) $r_{-1}$ in the $(k L, \theta)$-plane at $d / a=0.4$ and $\varepsilon_{r}=5.8$, dashed line-angle independent regime of $r_{0} \approx r_{-1} \approx 0.5$, dashed-dotted line-angle independent regime of $r_{-1} \approx 1$, dotted line-approximate boundary of the stop band with $r_{0} \approx 1$, and (c), (d) $r_{0}$ (solid line), $r_{-1}$ (dashed line), and $R$ (dotted line) versus $\theta$ at (c) $k L=5.57$ and (d) $k L=5.58$. 
relevant spot type behavior of $r_{-1}$ is observed in Fig. 2(b) at $3.4<k L<4.2$, i.e., at the left boundary of the region of $r_{-1} \approx 1$. They contribute to neither wide-angle splitting nor wide-angle spatial filtering.

Figures 2(c) and 2(d) quantitatively demonstrate the angle-tolerant splitting capability of the grating at fixed $k L . r_{0} \approx r_{-1} \approx 0.5$ remains almost constant in the $\theta$ interval that extends nearly from $10^{\circ}$ to $70^{\circ}$. Accordingly, $\phi_{-1}$ varies nearly from $-70^{\circ}$ to $-10^{\circ}$, i.e., this interval includes the Littrow mount regime. In turn, the angle between the incident and reflected beams varies from $0^{\circ}$ to nearly $60^{\circ}$.

As long as the reflection mode is considered, it can be possible to replace the regular (noncorrugated) part of the $\mathrm{PhC}$ grating with a properly designed one-dimensional Bragg mirror or a metallic mirror. Hence, one can substantially decrease the total thickness of the structure that is often important for practical applications. For simulation purposes, we used a thick metallic slab. Its permittivity is assumed to be given by $\varepsilon=1-\omega_{p}^{2} /[\omega(\omega-i \gamma)]$, where $\omega_{p}$ and $\gamma$ are plasma and collision frequency, respectively, with $\omega_{p} a / c=20 \pi$ and $\gamma / \omega_{p}=0.01$. Therefore, some losses are introduced compared to the structures in Figs. 1 and 2 . For example, for $\mathrm{Ag}$, we have $\omega_{p} /(2 \pi)=2.18 \times 1 \overline{0}^{15} \mathrm{~Hz}$, so that $a=1.376 \mu \mathrm{m}$ and, hence, $k L=4.3$ corresponds to $\lambda \approx 4 \mu \mathrm{m}$. It is noteworthy that the way of introduction of a reflector is ambiguous due to the freedom in the choice of the distance between it and the remaining rod layer. The distance between the rod center and metal surface is denoted by $D$, see Fig. 3 . Up to 10 values of $D$ have been compared, by keeping $D+h=3 a / 2$ with $h$ the metallic slab thickness.

Surprisingly, it was found that not only the use of a metallic slab can enable nearly the same response of zero (a)
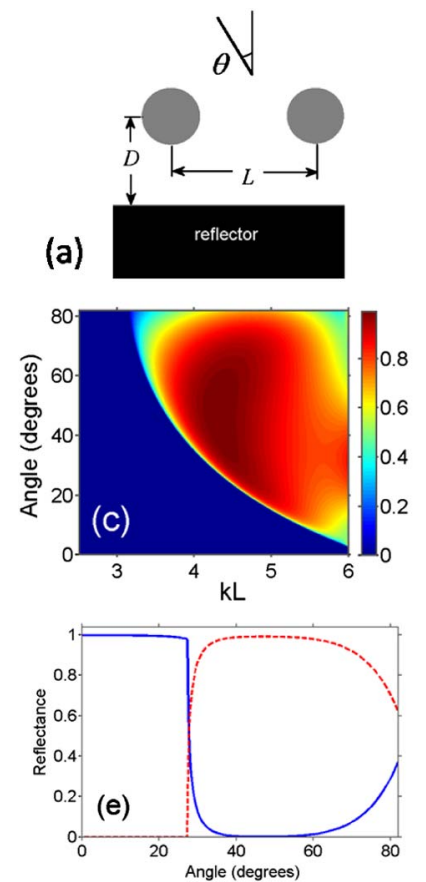
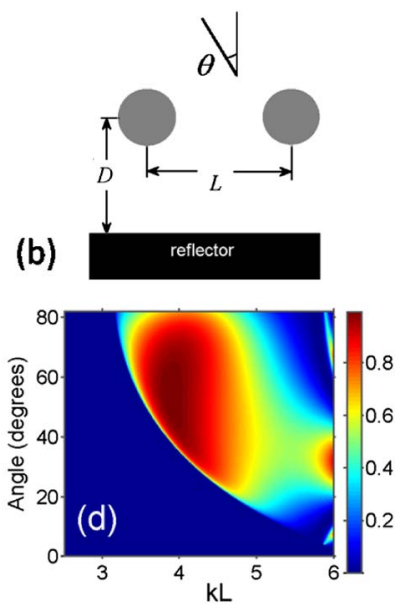

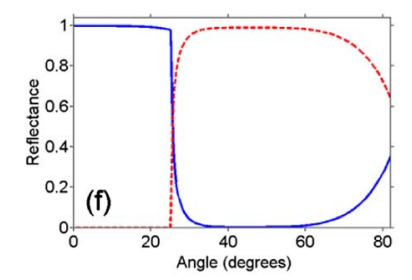

Fig. 3. Schematic of the single-layer rod grating over a metallic slab $(L=2 a)$ at (a) $D=0.775 a$ and (b) $D=a ; r_{-1}$ in the $(k L, \theta)$ plane (c) for configuration in case (a), and (d) for configuration in case (b), at $d / a=0.45$ and $\varepsilon_{r}=9.61$; and (e), (f) $r_{0}$ (solid line) and $r_{-1}$ (dashed line) versus $\theta$ for case (c), at (e) $k L=4.3$ and (f) $k L=4.4$. and first negative orders as the $\mathrm{PhC}$ grating in Fig. 1, but also variations in distance can exert a weak effect on the $\theta$-dependence of $r_{0}$ and $r_{-1}$. Figure 3 shows the results for two selected values of $D$. The change of $D$ results in a strong modification of the map of $r_{-1}$ in the $(k L, \theta)$-plane. In spite of this, the wide ranges of variations in $k L$ and $\theta$ with $r_{-1} \approx 1$, which are needed for wideband spatial filtering, are achieved for the both values of $D$. The width of the $k L$-range, in which $r_{-1}>0.95$, exceeds 0.45 and 0.25 (in units of $k L$ ) in Figs. 3(c) and 3(d), respectively, for $\theta$ varying at least between $50^{\circ}$ and $60^{\circ}$. The $k L$-values, at which the widest $\theta$ interval is obtained for $r_{-1}>0.95$, can significantly differ, as shown by comparison of Figs. 3(c) and $3(\mathrm{~d})$. Thus, by varying $D$, one changes the spectral location and width of the $\theta$ interval, in which $r_{-1}>r_{l}\left(r_{l}\right.$ is a minimal acceptable first-order efficiency). In terms of geometry, the structures in Fig. 3 are similar to those studied earlier for other operation regimes [ $\underline{9}-\underline{11}, \underline{24}]$.

For further evidence of the switching between the orders $m=0$ and $m=-1, r_{0}$ and $r_{-1}$ versus $\theta$ are shown in Figs. 3(e) and 3(f) for $D=0.775 a$. The agreement between Figs. 1(c) and 3(e) and between Figs. 1(d) and 3(f) is very good, although some imperfectness of reflection appears in Figs. 3(e) and 3(f) due to the Ohmic losses in the reflector.

Very recently, the first part of new simulations has been finished that are aimed to design performances for $\lambda=1 \mu \mathrm{m}$, which can be easily fabricated. In these designs, conventional optical materials are utilized, which are appropriate for the use of layer-by-layer lithography. $\mathrm{Au}$ can be used as the material for the reflector and rods that have now square or rectangular cross section, while $\varepsilon=2.25$ for the substrate. In particular, theoretical performances were found, in which $r_{-1}>0.7$ in a wide range of $\theta$ variation (e.g., $\Delta_{\theta}>30^{\circ}$ ). In the configurations with the same substrate, square rods, and the same remaining parameters as in Fig. $3, r_{-1}>0.92$ has recently been obtained for $\Delta_{\theta}>60^{\circ}$ at $\lambda=4 \mu \mathrm{m}$. For the transmission mode spatial filtering in the noncorrugated $\mathrm{PhC}$, efficiency near $100 \%$ in a large $\theta$ interval is attributed to the special case of Fabry-Perot type interference [3]. Here, $r_{-1} \approx 1$ in a large $\theta$ interval might be produced by a special case of Gires-Tournois interference between the grating and reflector [25]. Development of a proper theoretical framework is in progress.

In contrast to the spatial filtering in Figs. $\underline{1}$ and $\underline{3}$, replacement of the regular part of the $\mathrm{PhC}$ grating in Fig. $\underline{2}$ with a metallic reflector in the splitting regime is connected with the strong dependence of $r_{-1} / r_{0}$ on $D$. It is expected that a peculiar behavior of the impedance of the reflector surface is required to keep $r_{0}=r_{-1}=0.5$ in a wide range of $\theta$ variation. However, it can be a challenging task for $\theta$ varying, say, from $10^{\circ}$ to $70^{\circ}$.

To summarize, we demonstrated a route toward nearly perfect, high-/band pass, reflection mode spatial filtering for wide ranges of angles and incident-wave frequencies. The approach fundamentally relies on the capability of the single-layer rod grating to split the energy between the first and zero orders in a controllable way. In fact, it exploits the common effect of a wideband, (nearly) perfect reflector and a single-layer rod grating, which works as a switching element between zero and first negative orders. The reflector performances based on a slab of 
the PhC working in the lowest stop band and a thick metallic slab are shown to be identical in the sense of the filtering relevant reflection features. In particular, the suggested structures enable a nearly perfect blaze in wide ranges of parameter variation. The structures with a metallic reflector are crucial for possible replacement of bulky optical components by ultrathin planar ones. The reflection mode dual-beam diffraction splitter is demonstrated in the same $\mathrm{PhC}$ grating as that enabling spatial filtering. Specific behavior of the reflected diffraction orders at the edge of the PhC stop band allows us obtaining the dual-beam, equal-splitting regime for a wide incidence angle range. Design of spatial filters based on $3 \mathrm{D}$ structures for solid angle filtering is a more complex but solvable problem.

This work is supported by the projects DPT-HAMIT, ESF-EPIGRAT, and NATO-SET-181, and by TUBITAK under the Project Nos. 107A004, 109A015, and 109E301. A. E. S. thanks TUBITAK for partial support in the framework of the Visiting Researcher Program. E. O. acknowledges partial support from the Turkish Academy of Sciences.

\section{References}

1. L. Dettwiller and P. Chavel, J. Opt. Soc. Am. A 1, 18 (1984).

2. D. Schurig and D. R. Smith, Appl. Phys. Lett. 82, 2215 (2003).

3. A. E. Serebryannikov, A. Y. Petrov, and E. Ozbay, Appl. Phys. Lett. 94, 181101 (2009).

4. E. Colak, A. O. Cakmak, A. E. Serebryannikov, and E. Ozbay, J. Appl. Phys. 108, 113106 (2010).

5. R. Pico, I. Perez-Arjona, V. J. Sanchez-Morcillo, and K. Staliunas, Appl. Acoust. 74, 945 (2013).

6. V. Purlys, L. Maigyte, D. Gailevicius, M. Peckus, M. Malinauskas, and K. Staliunas, Phys. Rev. A 87, 033805 (2013).

7. O. F. Siddiqui and G. Eleftheriades, J. Appl. Phys. 99, 083102 (2006).
8. A. Sentenac and A.-L. Fehrembach, J. Opt. Soc. Am. A 22, 475 (2005).

9. B.-H. Cheong, O. N. Prudnikov, E. Cho, H.-S. Kim, J. Yu, J.-S. Cho, H.-J. Choi, and S. T. Shin, Appl. Phys. Lett. 94, 213104 (2009).

10. E. H. Cho, H.-S. Kim, B.-H. Cheong, O. Prudnikov, W. Xianyua, J.-S. Sohn, D.-J. Ma, H.-J. Choi, N.-C. Park, and Y.-P. Park, Opt. Express 17, 8621 (2009).

11. S. Steiner, S. Kroker, T. Kaesebier, E.-B. Kley, and A. Tuennermann, Opt. Express 20, 22555 (2012).

12. I. Moreno, J. J. Araiza, and M. Avedano-Alejo, Opt. Lett. 30, 914 (2005).

13. V. Purlys, L. Maigyte, D. Gailevicius, M. Peckus, M. Malinauskas, R. Gadonas, and K. Staliunas, Opt. Lett. 39, 929 (2014).

14. B. T. Schwartz and R. Piestun, J. Opt. Soc. Am. B 20, 2448 (2003).

15. C. Rizza, A. Ciattoni, E. Spinozzi, and L. Colombo, Opt. Lett. 37, 3345 (2012).

16. C. Ribot, M.-S. L. Lee, S. Collin, S. Bansropun, P. Plouhinec, D. Thenot, S. Cassette, B. Loiseaux, and Ph. Lalanne, Adv. Opt. Mat. 1, 489 (2013).

17. M.-S. L. Lee, Ph. Lalanne, J. C. Rodier, and E. Cambril, Opt. Lett. 25, 1690 (2000).

18. Ph. Lalanne, J. Hazart, P. Chavel, E. Cambril, and H. Launois, J. Opt. A Pure Appl. Opt. 1, 215 (1999).

19. L. Li and J. Hirsh, Opt. Lett. 20, 1349 (1995).

20. J. Zheng, C. Zhou, J. Feng, H. Cao, and P. Lu, Opt. Commun. 282, 3069 (2009).

21. A. E. Serebryannikov, A. O. Cakmak, and E. Ozbay, Opt. Express 20, 14980 (2012).

22. E. Colak, A. E. Serebryannikov, A. O. Cakmak, and E. Ozbay, Appl. Phys. Lett. 102, 151105 (2013).

23. T. Magath and A. E. Serebryannikov, J. Opt. Soc. Am. A 22, 2405 (2005).

24. M. Lester and D. C. Skigin, J. Opt. A Pure Appl. Opt. 9, 81 (2007).

25. P. Tournois, IEEE J. Quantum Electron. 33, 519 (1997). 